\title{
Etude sur modèle réduit du comportement d'une paroi de soutènement
}

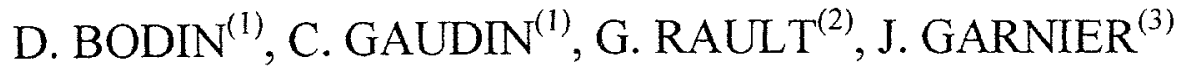 \\ (1) doctorants, (2) technicien supérieur, (3) chef de la division RMS \\ Laboratoire Central des Ponts et Chaussées \\ Route de Bouaye - B.P. 4129 \\ 44341 BOUGUENAIS Cedex
}

\begin{abstract}
Résumé :
Une première étude de faisabilité a été effectuée sur un modèle réduit en centrifugeuse. L'objectif est de reproduire la réalité du chargement d'un ouvrage de soutènement en cours d'excavation. Dans ce but, la simulation des phases de terrassement est réalisée à l'aide d'un robot sans arrêt de la centrifugeuse grâce à la mise en cuvre d'un nouvel équipement à ce jour unique. L'étude expérimentale présentée a permis la mesure continue des déplacements et des moments fléchissants d'une paroi de soutènement en cours d'excavation. L'essai a montré de grandes potentialités et permet d'envisager des études plus approfondies.
\end{abstract}

\begin{abstract}
:
A centrifuge model test has been run to study the behaviour of an embedded cantilever retaining wall. A boarded robot simulates stages of excavation, and the experiment device allows to measure the wall displacements and bending moments. This first feasibility test has been successful and a new series of more detailed experiments has been scheduled.
\end{abstract}

\section{Introduction}

Les études expérimentales sur modèles réduits centrifugés sont devenues une pratique relativement courante dans les recherches sur le comportement des ouvrages géotechniques ou lors du dimensionnement d'ouvrages exceptionnels. Les exemples d'application sont nombreux et concernent principalement les fondations sous charges statiques, cycliques ou dynamiques, les ancrages, les ouvrages souterrains, le renforcement de sol, le transport de polluants.

Pour étendre encore les domaines d'utilisation, un nouveau moyen d'essai a été récemment mis en service sur la centrifugeuse du Laboratoire Central des Ponts et Chaussées (LCPC). Il s'agit d'un robot équipé d'un changeur d'outils et fonctionnant sous des accélérations de $100 \mathrm{~g}$ (100 fois la pesanteur terrestre). Ce téléopérateur permet d'enchaîner différentes interventions sur le modèle, sans arrêt de la centrifugeuse. L'une des premières applications décrite ci-après concerne l'étude expérimentale du comportement de parois de soutènement où le robot a été utilisé pour effectuer les phases successives de terrassement, en alternance avec des sondages au pénétromètre statique. 


\section{Présentation du moyen d'essai}

Les essais sur modèles réduits ont nécessité le développement de matériels embarqués dans les centrifugeuses permettant d'effectuer les opérations pendant la centrifugation (dispositifs de chargement des ouvrages, de fonçage ou de battage de pieux, d'essais au pénétromètre et au scissomètre). Ces dispositifs assurent une seule fonction et sont le plus souvent installés à poste fixe [Garnier et al, 1999]. Un matériel plus polyvalent vient d'être mis en service au LCPC. Il s'agit d'un robot conçu par Rol-Cybernetics pour la partie contrôle-commande et par Acutronic (aujourd'hui Actidyn) pour l'ensemble mécanique. Sa tête peut se déplacer dans les trois directions au-dessus du conteneur d'essai et elle dispose aussi d'un degré de liberté en rotation. Le pilotage est effectué au moyen d'une commande numérique Robonum 800 (Télémécanique). La tête est équipée d'une interface universelle permettant de connecter différents outils préalablement installés dans un magasin (figure 1). Des connexions électriques et fluides (eau, air, huile) assurent l'alimentation éventuelle des outils et l'acquisition des mesures faites par les capteurs pouvant équiper ces outils. Ce robot et ses moyens annexes onit été décrits en détail par Derkx et al [1998] et Garnier et al [1999].

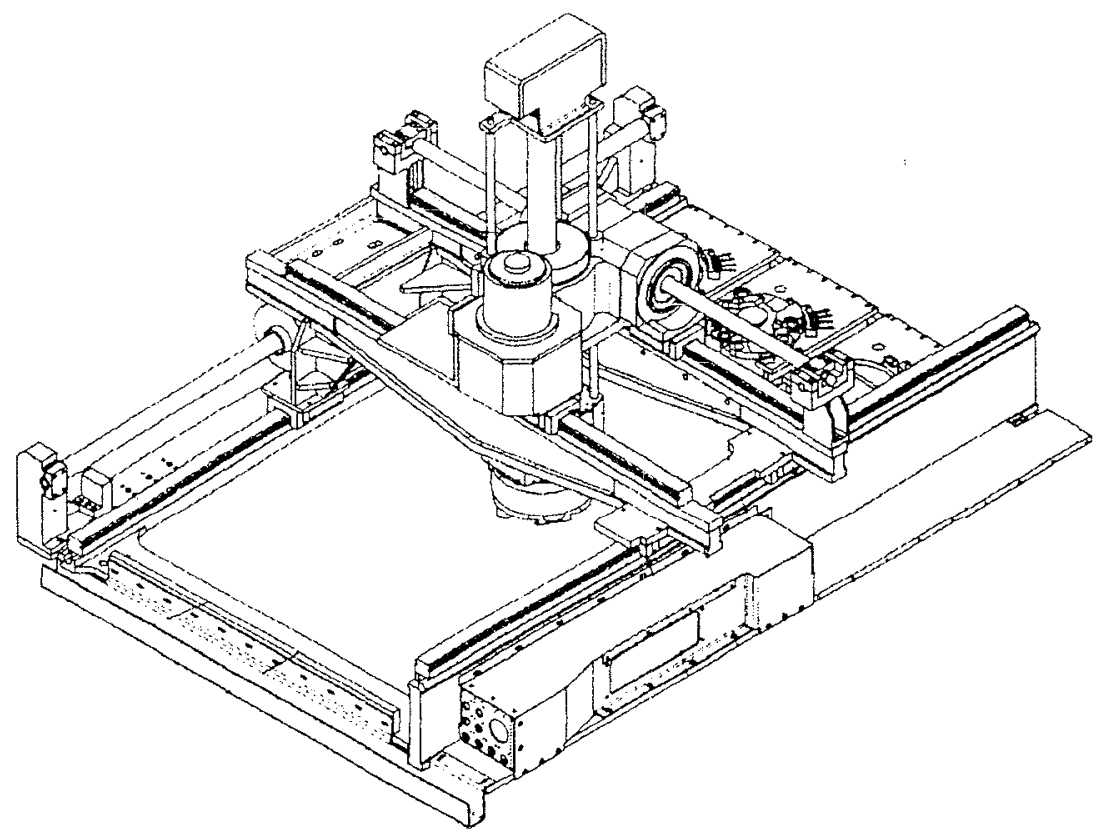

Figure 1 - Robot embarqué sur la centrifugeuse du LCPC. On-board robot on $L C P C$ centrifuge.

Les premières applications ont, jusqu'à présent, concerné des essais d'arrachement d'inclusions [Le Collinet, 1998], la réalisation de cartographies de résistance de pointe au pénétromètre [Ternet, 1999] et l'étude de faisabilité présentée ci-après portant sur le comportement de parois de soutènement [Bodin, 1999]. 


\section{Dispositif expérimental}

La modélisation physique en centrifugeuse est basée sur des lois de similitude entre les grandeurs physiques pour l'ouvrage à échelle 1 appelé prototype et pour l'ouvrage en modèle réduit. La spécificité du comportement des sols conduit à l'utilisation d'un sol réel sollicité dans le même domaine de contraintes. Ce choix conditionne l'augmentation des forces de masse sur le modèle réduit. On retient que pour un modèle réduit à une échelle $1 / \mathrm{n}$ son comportement devra être étudié sous une accélération de $\mathrm{n} * \mathrm{~g}$.

\subsection{Fabrication du modèle}

Les modèles réduits sont installés dans des conteneurs métalliques rigides, de dimensions intérieures $1,2 \times 0,80 \times 0,36 \mathrm{~m}$, qui sont ensuite transportés dans la nacelle de la centrifugeuse pour l'essai.

\subsubsection{Massif de sol}

Le massif de sol est constitué de sable de Fontainebleau sec. Le remplissage est effectué par pluviation, technique permettant la fabrication reproductible de massif relativement homogène à densité contrôlée. Le sol obtenu a un poids volumique $\gamma_{\mathrm{d}}=16 \mathrm{kN} / \mathrm{m}^{3}$ correspondant à un indice de densité $\mathrm{I}_{\mathrm{D}}=71 \%$.

A cette densité, l'angle de frottement mesuré par quatre essais à la boîte de cisaillement est de $36,5^{\circ}$ pour une cohésion de quelques $\mathrm{kPa}$.

\subsubsection{Paroi modèle réduit}

Le modèle réduit de paroi a une fiche initiale de $200 \mathrm{~mm}$. Ce modèle au $1 / 70^{\text {ème }}$ testé sous une accélération de $70 \mathrm{~g}$ est constitué de plaques d'alliage d'aluminium AU4G de $5 \mathrm{~mm}$ d'épaisseur. Deux cellules de garde ont été disposées de part et d'autre de la cellule de mesure afin d'éliminer les effets de bord dus aux faces du conteneur, le but étant de simuler un comportement en déformation plane.

\subsubsection{Ouvrage prototype modélisé}

L'ouvrage modélisé ìci à l'échelle $1 / 70$, est une paroi de $14 \mathrm{~m}$ de hauteur. Sa rigidité en flexion équirvaut à une paroi moulée en béton armé de $0,6 \mathrm{~m}$ d'épaisseur.

\subsection{Instrumentation}

Afin de suivre l'évolution de la paroi en cours de terrassement, le modèle réduit est équipé de (fig. 2):

4 capteurs potentiométriques mesurant les déplacements de la paroi à trois profondeurs différentes;

10 paires de jauges montées en demi-pont mesurant les déformations de flexion de la paroi ;

- 2 capteurs potentiométriques mis en place derrière le rideau pour mesurer les tassements du massif amont. 
Les mesures de déformation de la paroi par jauges extensométriques permettent de déterminer les moments fléchissants. Un calibrage sous une sollicitation de flexion a permis de vérifier initialement le bon comportement des jauges.

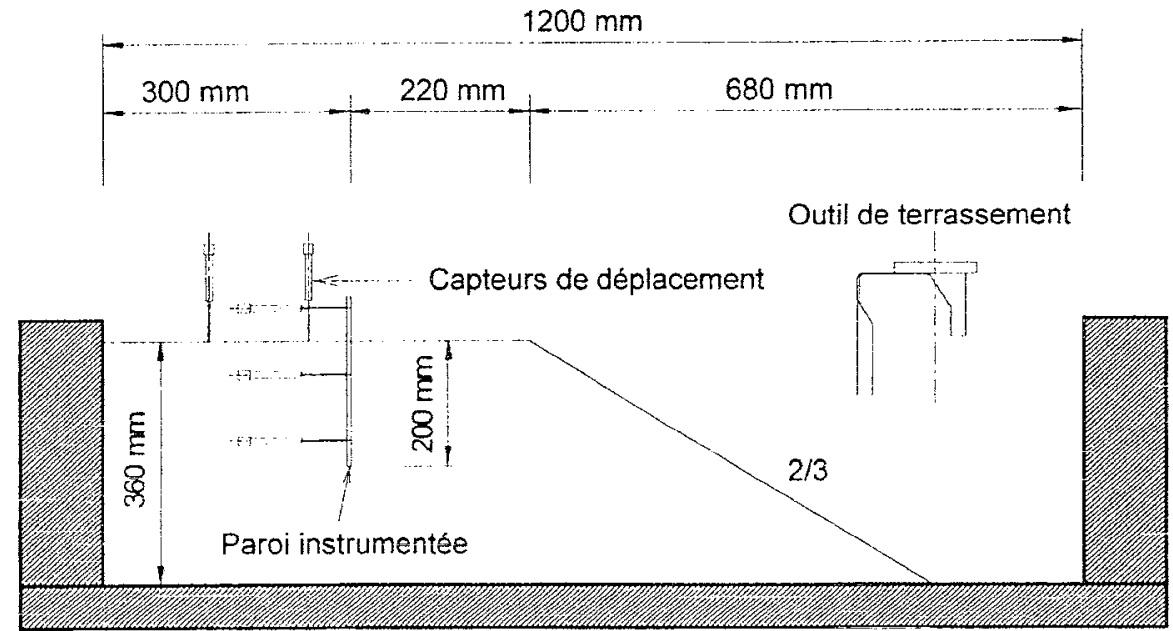

Figure 2 : schéma du disp̄ossitif expérimental.

Used experimental device.

\subsection{Programme de chargement}

Le déroulement de l'essai consiste à excaver progressivement la fouille devant la paroi. Le robot embarqué reproduit un programme de nivellement du talus par passes successives dont l'épaisseur décroît de $3 \mathrm{~mm}$ à $1 \mathrm{~mm}$ en fin d'essai.

Le mode de réalisation choisi pour l'excavation nécessite la présence d'un talus dans le conteneur pour le stockage des déblais (fig. 3). Ce dispositif complique les interprétations car sa géométrie est variable en fonction du terrassement. On verra également qu'il influence l'état de contrainte initial dans le massif $(\S 5.1)$.
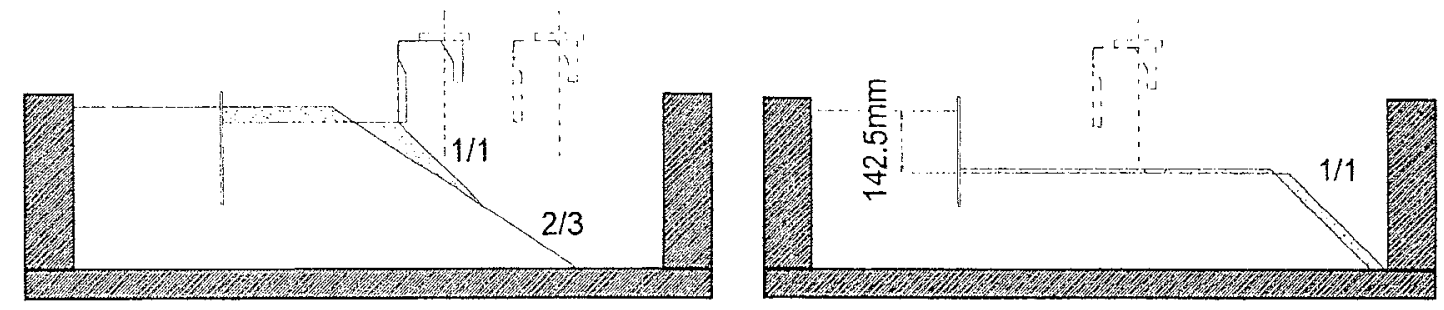

Figure 3 : Evolution de la géométrie du massif entre le début et la fin de l'essai. Soil geometry evolution during the test 


\section{Premiers enseignements}

\subsection{Données sur le comportement de la paroi}

\subsubsection{Résultats expérimentaux}

Les mesures de déplacements horizontaux de la paroi et de tassements de la surface du massif amont sont présentées (fig. 4-a) en fonction de l'avancement de l'excavation. Ce dernier sera caractérisé par le rapport d'excavation $\mathrm{H}_{\mathrm{L}} / \mathrm{H}$ (rapport de la hauteur excavée $\mathrm{H}_{\mathrm{L}}$ à la hauteur totale $\mathrm{H}$ de la paroi).

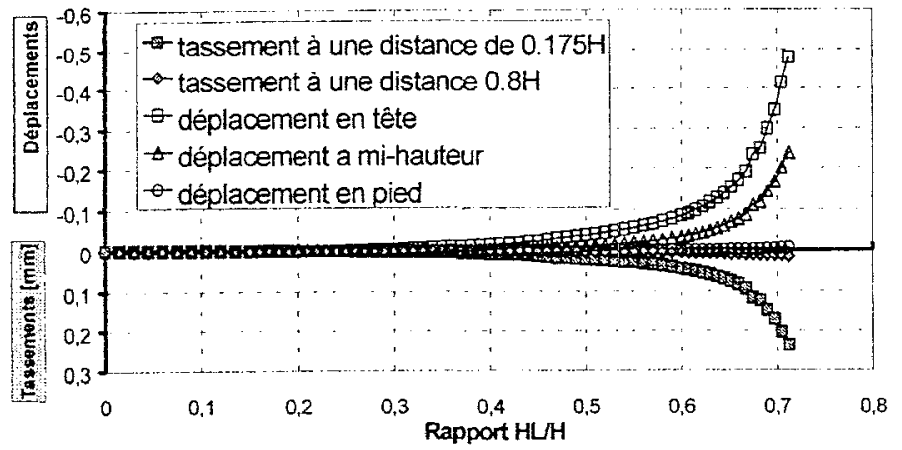

$-a-$

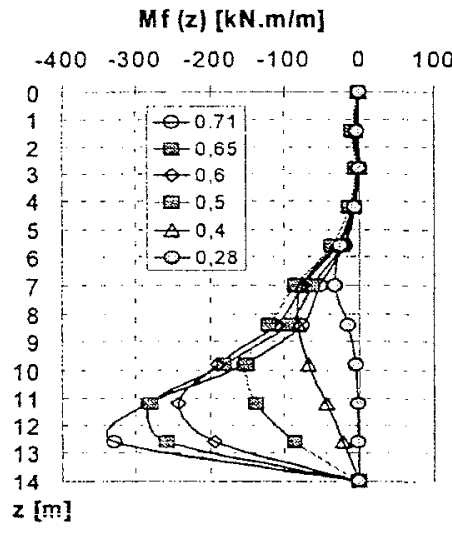

$-b-$

Figure 4 : Déplacements de la paroi, tassements du massif amont (a) et moments fléchissants (b) en fonction du rapport d'excavation $\mathrm{H}_{\mathrm{L}} / \mathrm{H}$ [Bodin, 1999]. Wall displacements, soil settlement (a) and bending moments for differents excavation ratio.

La figure 4-b montre les courbes de moments fléchissants développés dans la paroi en fonction de la profondeur pour différents rapports d'excavation (fig. 4-b). Les variations observées (maxima et points d'inflexions) sont cohćrentes avec la mobilisation progressive de pressions de poussée et de butée.

\subsubsection{Premières comparaisons}

L'étude des écrans de soutènement peut être menée avec différents degrés de complexité. Ces premiers résultats ne portent que sur un seul essai et doivent être considérés avec quelques précautions. La théorie des équilibres de poussée et butée limites donne une première approximation sur les sollicitations proches de la rupture. Cette méthode conduit, pour une paroi autostable dans un sable sec $\left(\gamma=16 \mathrm{kN} / \mathrm{m}^{3}, \varphi=36,5^{\circ}, \mathrm{c}=0\right)$, aux valeurs suivantes [Houy, 1976] :

- rapport d'excavation $\mathrm{H}_{\mathrm{L}} / \mathrm{H}$ limite de 0,67 ;

- moment de flexion maximal $\mathrm{M}_{\max }=650 \mathrm{kN} . \mathrm{m} / \mathrm{m}$.

$\mathrm{Si}$ on considère les déplacements du rideau comme représentatifs $\mathrm{du}$ comportement global de la paroi, le rapport d'excavation limite calculé est du même ordre de grandeur que celui qui a été observé (figure 4-a). Le moment maximal mesuré (\#330 $\mathrm{kN} . \mathrm{m} / \mathrm{m}$ ) est inférieur de moitié à la valeur limite 
théorique. Cette différence s'explique sans doute en partie par l'hypothèse d'une cohésion nulle faite dans le calcul théorique.

\subsection{Evaluation du dispositif expérimental}

Le dispositif utilisé avait pour but de s'assurer de la faisabilité technique d'une excavation en centrifugeuse et de la validité de la modélisation physique d'écrans de soutènement. En ce sens, il a bien rempli son rôle. Néanmoins, différents points nécessitent d'être améliorés pour pouvoir mener plus en avant les études sur le comportement des écrans de soutènement.

La présence du talus en amont de l'écran engendre une modification de l'état de contraintes initiales qu'il y a lieu d'étudier et, au besoin, de circonscrire. En outre ce talus et le mode de stockage des déblais diminuent les dimensions du modèle et imposent des conditions aux limites qui peuvent ne pas être tout à fait réalistes.

L'écran de soutènement s'est montré trop rigide et n'a pas permis une mesure précise des moments dans les parties peu sollicitées ainsi que dans les premières phases d'excavation.

La méthode d'excavation retenue est satisfaisante. L'épaisseur des couches terrassées et la vitesse d'avancement de l'outil simulent correctement une excavation réelle.

Ces différentes remarques nous ont amené à modifier le dispositif expérimental comme indiqué au chapitre suivant.

\section{Evolutions et perspectives}

\subsection{Le massif de sol}

Un doute subsistait quant à l'effet du talus sur l'état de contraintes initiales dans le massif et sur le comportement général de l'écran. Des mesures de contraintes horizontales dans un massif centrifugé à différentes distances de la crête du talus ont été réalisées avec la méthode développée par Ternet [1999]. Les données expérimentales et les résultats des simulations numériques avec le progiciel CESAR- LCPC ont toutes deux montré une diminution générale de l'état de contraintes de l'ordre de $20 \%$ à l'aplomb de la crête du talus. Cette diminution est ressentie jusqu'à une distance de $20 \mathrm{~m}$ prototype (fig. 5).

L'influence directe du talus et de cette diminution des états de contraintes initiales sur le comportement de la paroi est difficile à estimer. Cependant, dans un souci de meilleure représentativité et de meilleure mâtrise de la modélisation, il convient de minimiser cette influence.

Le dispositif expérimental a donc été modifié (fig. 6). Le talus est désormais tronqué et sa pente est raidie grâce à l'inclusion de plusieurs lits de géotextiles. 

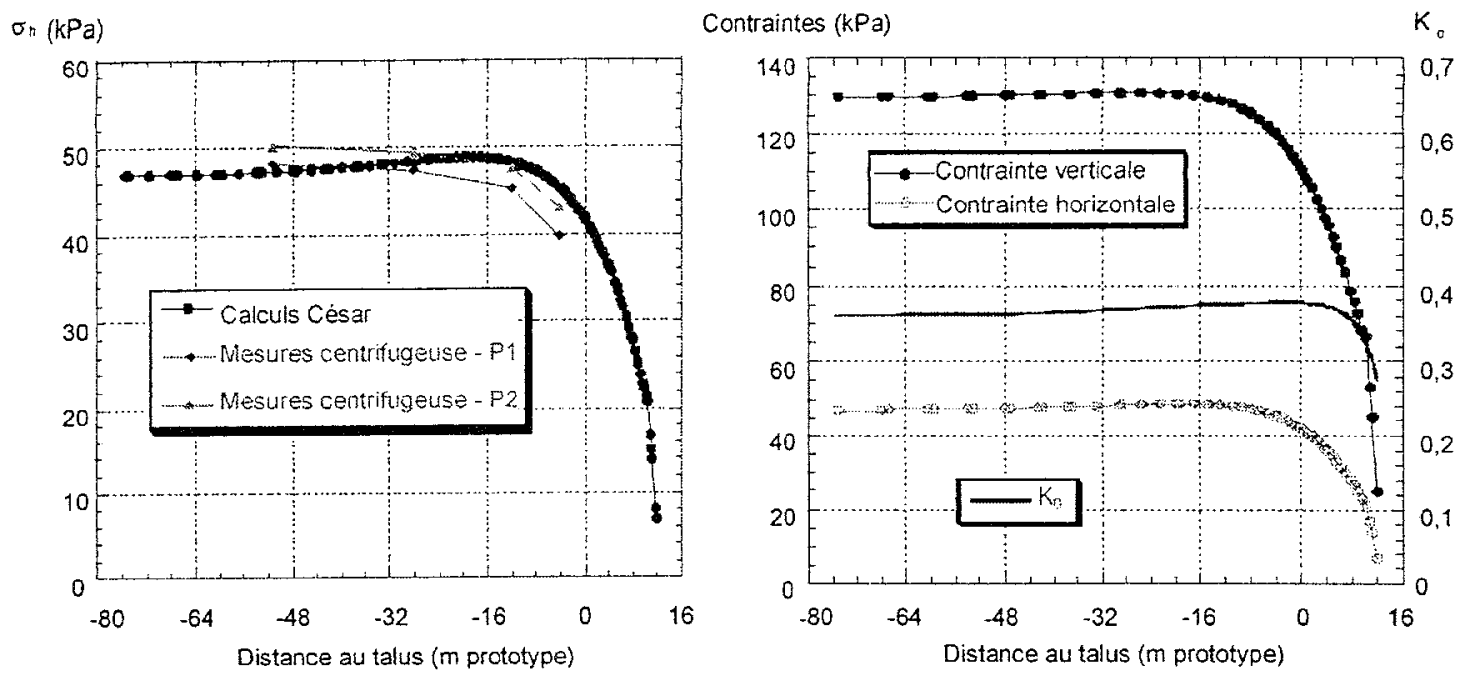

Figure 5 - Influence du talus sur l'état de contrainte initial.

Slope influence on the initial stress state.

Il a par ailleurs semblé nécessaire d'augmenter la surface utile du dispositif en

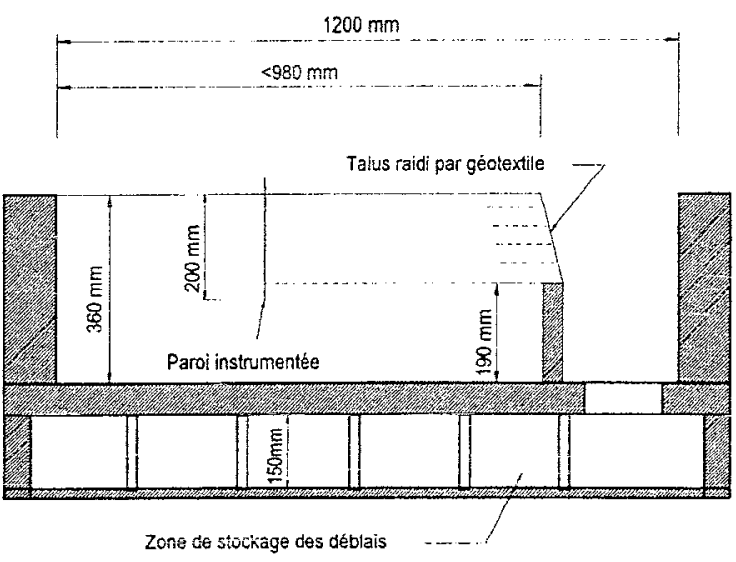

Figure 6 - Schéma de principe du second dispositif expérimental

New assumed experimental device repoussant au maximum les frontières du massif. Pour ce faire, un faux fond a été mis en place (fig 6) afin de stocker les déblais soüs le massif, et non plus devant celui-ci comme précédemment. Ce faux fond associé à l'éloignement du talus permet d'augmenter la longueur du massif de $520 \mathrm{~mm}$ à $980 \mathrm{~mm}$.

Les conditions de déformations planes sont assurées par la mise en place de celluies de garde comme précédemment.

\subsection{L'écran de soutènement}

Le premier essai a permis de préciser les caractéristiques que devraient avoir l'écran modèle pour une bonne modélisation.

La paroi doit être suffisamment souple pour assurer une déformabilité suffisante des fibres extrêmes et ainsi une meilleure précision sur les moments de flexion. L'instrumentation doit en outre être suffisamment importante de façon notamment à disposer de plus d'informations dans les zones de butée où se situent les forts gradients de moments.

Le second modèle mis en œuvre sera donc une paroi en aluminium AU4G de $240 \mathrm{~mm}$ de hauteur ( $200 \mathrm{~mm}$ en fiche) pour $2 \mathrm{~mm}$ d'épaisseur, ce qui constitue un 
bon compromis entre la déformabilité et la représentativité du prototype (palplanches de type Larssen SL4). Elle sera instrumentée de 22 paires de jauges de déformations espacées de $10 \mathrm{~mm}$. Les mesures discrètes de moments pourront permettre après double dérivation et double intégration selon la théorie des poutres, d'avoir accès aux déplacements de l'écran et aux pressions résultantes s'exerçant sur celui-ci.

\section{Conclusions}

Ces premiers essais ont démontré la faisabilité d'études sur modèles centrifugés du comportement des parois de soutènement (excavation en cours de centrifugation, mesures des efforts et des déplacements). Si la profondeur d'excavation limite observée est en accord avec les prévisions des méthodes d'équilibre limite, il n'en est pas tout à fait de même avec les moments maximums. Ce point sera étudié lors des prochaines expériences.

Avec le nouveau dispositif expérimental qui sera opérationnel au premier trimestre 2000, diverses configurations de soutènement pourront être étudiées (parois butonnée, multiancrée ...), ainsi que plusieurs phénomènes directs ou annexes (phasage de réalisation, mise en tension des tirants, mécanismes de rupture, interaction aux ouvrages environnants...). Ces différentes recherches font l'objet d'un travail de thèse qui prendra fin le dernier trimestre 2002.

\section{Références bibliographiques}

Bodin D. (1999), Modélisation physique des parois de soutènements - Etude de faisabilité, Rapport de DEA, Université de Nantes, $76 \mathrm{p}$.

Derkx F., Merliot E., Garnier J., Cottineau L.M. (1998), On-board remotecontrolled centrifuge robot, Centrifuge 98, Tokyo, Balkema, pp. 97-102.

Garnier J., Derkx F., Cottineau L.M., Rault G. (1999), Etudes géotechniques sur modèles centrifugés - Evolution des matériels et des techniques expérimentales, Bulletin des Laboratoires des Ponts et Chaussées, $\mathrm{n}^{\circ} 223$, Septembre-Octobre, $24 \mathrm{p}$.

Houy A. (1976), Calcul des ouvrages en palplanche métallique, Edité par Sacilor, $115 \mathrm{p}$.

Le Collinet J. (1998), Effets de taille et d'accélération dans le cisaillement des interfaces sol/pieu, Rapport de DEA, Université de Nantes, $85 \mathrm{p}$.

Ternet O. (1999), Reconstitution et caractérisation des massifs de sable: application aux essais en centrifugeuse et en chambre de calibration, Thèse de doctorat, Université de Caen, $184 \mathrm{p}$. 